\title{
Potentials for High-Energy Scattering from Hydrogenlike Atoms*
}

\author{
J. F. Reading $\dagger^{\dagger}$ \\ Northeastern University, Boston, Massachusetts 02115 \\ (Received 3 November, 1969)
}

The time-dependent equation

$$
\frac{i \hbar v \partial \varphi(r, R)}{\partial Z}=\exp \left(i H_{e} Z / \hbar v\right) V(r, R) \exp \left(-i H_{e} Z / \hbar v\right) \varphi(v, R) \quad, \quad t=Z / v
$$

describing a fast particle perturbing a bound system was solved by Glauber in the diabatic approximation of setting $H_{e}$ equal to zero. The nonlocal potential $\exp \left(i H_{e} Z / \hbar v\right) V(r, R)$ $\times \exp \left(-i H_{e} Z / \hbar v\right)$ is thus approximated by a local or diagonal form $V(r, R)$. In this paper the local approximation is retained. The bound system is assumed to consist of a single electron attached to a fixed point with wave function $\exp \left(-r / a_{0}\right)$. It is then shown how the diabatic approximation can be relaxed by modifying $V$ to include the effect of $H_{e}$ to order $v^{-1}$. In particular in the impulse approximation, scattering is described by a static local potential $V_{L}(r, R)$ $=1 / R-\left[\operatorname{erf}\left(i^{1 / 2} \alpha+\epsilon\right)\right] /|\overrightarrow{\mathrm{R}}-\overrightarrow{\mathrm{r}}|$, where $\alpha^{2}=|\overrightarrow{\mathrm{R}}-\overrightarrow{\mathrm{r}}|^{2} /(2 c Z)$, and $c=\hbar /\left(m v a_{0}\right)$. An analytic form is given for $\varphi(r ; \overrightarrow{\mathrm{B}}, \infty)$. The binding of the electron neglected in the impulse approximation can be taken into account by changing $V_{L}$ to $V_{L}(r+x, R)$, where $x=e^{i c Z}-1$. The scattering problem is thus reduced to quadratures.

\section{INTRODUCTION}

It is the purpose of this paper to show the modification to the local potential necessary to relax the diabatic approximation ${ }^{1,2}$ for scattering from hydrogenlike systems. The main result is that we are able to do this to order $v^{-1}$ in a local approximation.

We consider the problem of a fast projectile (energy $E$, mass $m_{p}$, coordinate $\overrightarrow{\mathrm{R}}$ ) incident on an electron (mass $m_{e}$, coordinate $\overrightarrow{\mathrm{r}}$ ) bound to a fixed positively charged point located at the origin of our coordinate system. This problem contains the essential difficulties of electron-, muon-, or proton-hydrogen scattering at high energies. The wave equation describing the motion is

$$
\left[-\left(\hbar^{2} / 2 m_{p}\right) \nabla_{R}^{2}+H_{e}+V(r, R)\right] \psi=E \psi,
$$

where $H_{e}=-\left(\hbar^{2} / 2 m_{e}\right) \nabla_{r}^{2}-e^{2} / r-W_{0}$

and $V(r, R)=e^{z}[(1 / R)-1 /|\overrightarrow{\mathrm{R}}-\overrightarrow{\mathrm{r}}|]$.

The complete set of eigenfunctions of $H_{e}$ satisfies the equation

$$
H_{e} \chi_{n}=W_{n} \chi_{n}, \quad H_{e} \chi_{0}=0
$$

and the electron is initially in its $1 s$ ground state $\chi_{0}$.

At sufficiently high energies the projectile can be considered to provide a time-dependent potential which perturbs the bound system from which it is scattering. ${ }^{2}$ The projectile is considered initially to be moving along the $Z$ axis at some impact parameter $\vec{B}$; we define $\varphi_{s}$ as

$$
\psi=e^{i k Z} \varphi_{s},
$$

where $k=\left(2 m_{p} E / \hbar^{2}\right)^{1 / 2}$ and derive

$$
\left[-\frac{\hbar^{2}}{2 m_{p}} \nabla_{R}^{2}-\frac{i k \hbar^{2}}{m_{p}} \frac{\partial}{\partial Z}+H_{e}+V(r, R)\right] \varphi_{s}=0 .
$$

If we are in the region of parameters where

$$
k R_{0} \gg 1, E \gg V,
$$

where $R_{0}$ is some measure of the range of $V(r, R)$, we can neglect the " $\nabla_{R}^{2}$ " term in Eq. (1) and derive the approximate equation ${ }^{2}$

$$
\frac{i \hbar v \partial \varphi_{s}}{\partial Z}=\left[H_{e}+V(r, R)\right] \varphi_{s} .
$$

In Eq. (3) $v$ is the initial velocity of the projectile. If we write $\vec{R}$ as $\vec{R}=(\vec{B}, Z)=(\vec{B}, v t)$, we see that Eq. (2) is the time-dependent Schrödinger equation. Glauber ${ }^{2}$ pointed out that if we are in the region of parameters given by Eq. (2), all the scattering takes place in the forward direction. The recoil energy $H_{e}$ of the bound particle is then presumably negligibly small. If we set $H_{e}$ equal to zero, i.e., make the diabatic approximation, Eq. (3) is soluble, and we obtain

$$
\varphi_{s}=\exp \left(-\frac{i}{\hbar v} \int_{-\infty}^{z} V\left(r ; \overrightarrow{\mathrm{B}}, Z^{\prime}\right) d Z^{\prime}\right) \chi_{0}(r) 。
$$

This argument given by Glauber with the nuclear problem in mind might be misleading in an atomic collision. In the nuclear case the mass of the projectile is equal to or less than the mass of the struck target particle and the range of the twobody force is less than the dimensions of the bound system. These conditions do not necessarily hold in an atomic collision, and it is not true that being in a situation where one can apply the high-energy approximation to two-body force generally implies 
that one is in a situation where the diabatic approximation will be good.

In this paper we show how to include the effect of $H_{e}$ to first order in $v^{-1}$. The "obvious" way to proceed is to substitute the approximate solution of Eq. (4) into Eq. (3) and iterate on the $H_{e}$ term. ${ }^{3}$ This unfortunately leads immediately to difficulties. (i) The term $\nabla_{r}^{2} V$ produces a $\delta$ function at $\overrightarrow{\mathrm{r}}=\overrightarrow{\mathrm{R}}$. (ii) The term $\vec{\nabla} \chi \cdot \vec{\nabla} V$ produces a divergence in the "potential" at large $Z$.

We have been able to get rid of the former difficulty but only to circumvent the latter by a choice of a cutoff which is not unique.

Before proceeding to the problem, it is well to consider what we need to calculate. One always has a choice in a time-dependent problem of working with $\varphi_{s}$ or $\varphi$ where

$$
\varphi=\exp \left(i H_{e} Z / \hbar v\right) \varphi_{s} .
$$

The equation for $\varphi$ is

$$
i \hbar \nu \frac{\partial \varphi}{\partial Z}=\exp \left(\frac{i H_{e} Z}{\hbar v}\right) v(r, R) \exp \left(\frac{-i H_{e} Z}{\hbar v}\right) \varphi .
$$

If we intend to calculate direct excitations of the bound system, we will be concerned with amplitudes such as

$$
\begin{aligned}
& f_{m, 0}\left(\overrightarrow{\mathrm{k}}_{m}, \overrightarrow{\mathrm{k}}\right)=-\frac{2 m_{p} k_{m}}{4 \pi \hbar^{2} k} \int d^{3} r d^{3} R \chi_{m}(r) V(r, R) \varphi_{s} \\
& \times \exp \left(-i \overrightarrow{\mathrm{k}}_{m} \cdot \overrightarrow{\mathrm{R}}+i k Z\right), \\
& \text { where } \quad k_{m}=\left[2 m_{p}\left(E-W_{m}\right) / \hbar^{2}\right]^{1 / 2} \text {. }
\end{aligned}
$$

For large $E$ we can expand the square root in Eq. (8). For small angle scattering we may then rewrite Eq. (8) as

$$
\begin{aligned}
& f_{m, 0}\left(\overrightarrow{\mathrm{k}}_{m}, \overrightarrow{\mathrm{k}}\right) \approx-\frac{m_{p}}{2 \pi \hbar^{2}} \int d^{3} r d^{3} R \chi_{m}(r) V(r, R) \varphi_{s} \\
& \quad \times \exp \left(-i \overrightarrow{\mathrm{k}}_{m} \cdot \overrightarrow{\mathrm{B}}+i W_{m} Z / \hbar v\right) \\
& =\frac{k}{2 \pi i} \int d^{3} R \exp (-i \overrightarrow{\mathrm{k}} \cdot \overrightarrow{\mathrm{B}}) \frac{\partial}{\partial Z}\left\langle\chi_{m} \mid \varphi(\overrightarrow{\mathrm{B}}, Z)\right\rangle \\
& =\frac{k}{2 \pi i} \int d^{2} \mathrm{~B} \exp \left(-i \overrightarrow{\mathrm{k}}_{m} \cdot \overrightarrow{\mathrm{B}}\right)\left[\left\langle\chi_{m} \mid \varphi(\overrightarrow{\mathrm{B}}, \infty)\right\rangle-\delta_{m 0}\right]_{0}
\end{aligned}
$$

Thus for direct excitations we need $\varphi(r ; \overrightarrow{\mathrm{B}}, \infty)$. On the other hand, for exchange processes we need $\varphi_{s}$. In the following we shall work with $\varphi$ giving the conversion to $\varphi_{s}$ where necessary.

\section{LOCAL IMPULSE APPROXIMATION}

In a system of units where lengths are measured in units of the Bohr radius $a_{0}$ we may rewrite Eq. (6) as

$$
\frac{\partial S}{\partial Z}=-i c \exp (-\bar{H} c Z)\left(\frac{1}{R}-\frac{1}{|\overrightarrow{\mathrm{R}}-\overrightarrow{\mathrm{r}}|}\right) \exp (-\bar{H} c Z),
$$

where $\varphi=\chi_{0}(r) S=e^{-r} S$

$$
\bar{H}=-\frac{1}{2} \nabla_{r}^{2}-\vec{\nabla} \ln \chi_{0} \cdot \vec{\nabla}_{r}=-\frac{1}{2} \nabla_{r}^{2}+\frac{\partial}{\partial r},
$$

and $\quad c=m_{p} /\left(m_{e} k a_{0}\right)$.

The condition for the diabatic approximation to hold is that $\bar{H} c Z \ll 1$, which implies that ${ }^{2}$

$$
\left(m_{p} / m_{e}\right)\left[O\left(1 / k R_{0}\right)+O(V / E)+O\left(1 / k a_{0}\right)\right] \ll 1 .
$$

To derive Eq. (12) we have assumed $S$ varies appreciably over some range $R_{0}$. Clearly the inequality of Eq. (12) is not guaranteed by Eq. (2).

We see that $\bar{H}$ in Eq. (11) splits naturally into two terms. We shall call the first the freely recoiling term (ft) and the latter the binding term (bt). This nomenclature expresses the fact that in the limit of zero binding energy bt is zero. It is the great simplification of the hydrogen system that bt is a constant times " $\partial / \partial r$." Exponentiated, this is just a shift operator.

We consider ourselves to be in a region of parameters where $c$ is small, and we want to calculate the correction to the diabatic approximation to first order in $c$. We write

$$
\begin{aligned}
\exp (i \bar{H} c Z)= & \exp \left(i c Z \frac{\partial}{\partial r}+\frac{(i c Z)^{2}}{r^{3}} \frac{\partial}{\partial r}+\frac{(i c Z)^{2}}{r 3} \nabla_{\phi, \varphi}^{2}+\cdots\right) \\
& \times \exp \left(-\frac{1}{2} i c Z \nabla_{r}^{2}\right) .
\end{aligned}
$$

In the impulse approximation we assume the struck target particle recoils freely which means we only keep the last exponential in Eq. (13). We are thus led to study the equation

$$
\frac{\partial S}{\partial Z}=-i c \exp \left(-\frac{i c Z}{2} \nabla_{r}^{2}\right) V(r, R) \exp \left(\frac{i c Z}{2} \nabla_{r}^{2}\right) S
$$

We solve this equation approximately by an expansion in terms of the nonlocality of the transformed potential. This differs from other authors' treatment of the impulse approximation. ${ }^{5}$

In a plane-wave representation we have

$$
\begin{aligned}
\frac{\partial s}{\partial Z}(\overrightarrow{\mathrm{k}}, \overrightarrow{\mathrm{R}})= & -i c \int d^{3} k_{2} \exp \left(\frac{i c Z}{2}\left(k_{1}^{2}-k_{2}^{2}\right)\right) \\
& \times v\left(\overrightarrow{\mathrm{k}}_{1}-\overrightarrow{\mathrm{k}}_{2}, \overrightarrow{\mathrm{R}}\right)_{s}\left(\overrightarrow{\mathrm{k}}_{2}, \overrightarrow{\mathrm{R}}\right),
\end{aligned}
$$

where $\quad s\left(\overrightarrow{\mathrm{k}}_{1}, \overrightarrow{\mathrm{R}}\right)=\int \exp \left(-i \overrightarrow{\mathrm{k}}_{1} \cdot \overrightarrow{\mathrm{r}}\right) S(\overrightarrow{\mathrm{r}}, \overrightarrow{\mathrm{R}}) \frac{d^{3} r}{(2 \pi)^{3 / 2}}$

$$
\begin{aligned}
v(\overrightarrow{\mathrm{q}}, \overrightarrow{\mathrm{R}}) & =\int \exp (-i \overrightarrow{\mathrm{q}}, \overrightarrow{\mathrm{r}}) V(r, R) \frac{d^{3} r}{(2 \pi)^{3 / 2}}, \\
& =\frac{\delta(q)}{R}-\frac{e^{-i \overrightarrow{\mathrm{q}} \cdot \overrightarrow{\mathrm{R}}}}{2 \pi^{2} q^{2}} .
\end{aligned}
$$

Returning to configuration space we obtain

$$
\frac{\partial s\left(\overrightarrow{\mathrm{r}}_{1}, \overrightarrow{\mathrm{R}}\right)}{\partial z}=-\frac{i c}{(2 \pi)^{3}} \int d^{3} k_{1} d^{3} k_{2} d^{3} r_{2}
$$




$$
\begin{aligned}
& \times \exp \left[\frac{1}{2} i c Z\left(k_{1}^{2}-k_{2}^{2}\right)+i \overrightarrow{\mathrm{k}}_{1} \cdot \overrightarrow{\mathrm{r}}_{1}-i \overrightarrow{\mathrm{k}}_{2} \cdot \overrightarrow{\mathrm{r}}_{2}\right] \\
& \times v\left(\overrightarrow{\mathrm{k}}_{1}-\overrightarrow{\mathrm{k}}_{2}, \overrightarrow{\mathrm{R}}\right) S\left(\overrightarrow{\mathrm{r}}_{2}, \overrightarrow{\mathrm{R}}\right) .
\end{aligned}
$$

Changing coordinates

$$
\overrightarrow{\mathrm{q}}=\overrightarrow{\mathrm{k}}_{1}-\overrightarrow{\mathrm{k}}_{2}, \quad 2 \overrightarrow{\mathrm{Q}}=\overrightarrow{\mathrm{k}}_{1}+\overrightarrow{\mathrm{k}}_{2},
$$

and integrating on $Q$ gives

$$
\begin{aligned}
\frac{\partial S\left(\overrightarrow{\mathrm{r}}_{1}, \overrightarrow{\mathrm{R}}\right)}{\partial Z}= & -i c \int d^{3} q d^{3} r_{2} \delta\left(\overrightarrow{\mathrm{r}}_{1}-\overrightarrow{\mathrm{r}}_{2}+Z \overrightarrow{\mathrm{q}}\right) \\
& \times \exp \left[\frac{1}{2} i \overrightarrow{\mathrm{q}} \cdot\left(\overrightarrow{\mathrm{r}}_{1}+\overrightarrow{\mathrm{r}}_{2}\right)\right] V(\overrightarrow{\mathrm{q}}, \overrightarrow{\mathrm{R}}) S\left(\overrightarrow{\mathrm{r}}_{2}, \overrightarrow{\mathrm{R}}\right) \\
= & -i c \int V_{N L}\left(\overrightarrow{\mathrm{r}}_{1}, \overrightarrow{\mathrm{r}}_{2}\right) S\left(\overrightarrow{\mathrm{r}}_{2}, \overrightarrow{\mathrm{R}}\right) d^{3} r_{2} .
\end{aligned}
$$

Therefore

$$
\begin{aligned}
S(\overrightarrow{\mathrm{r}}, \overrightarrow{\mathrm{R}})= & \exp \left(-i c \int_{-\infty}^{z} d Z^{\prime} \int d^{3} r^{\prime} S^{-1}\left(\overrightarrow{\mathrm{r}}, \overrightarrow{\mathrm{B}}, \overrightarrow{\mathrm{Z}}^{\prime}\right)\right. \\
& \left.\times V_{N L}\left(\overrightarrow{\mathrm{r}}, \overrightarrow{\mathrm{r}}^{\prime}\right) S\left(\overrightarrow{\mathrm{r}}^{\prime} ; \overrightarrow{\mathrm{B}}, \overrightarrow{\mathrm{Z}}^{\prime}\right)\right) .
\end{aligned}
$$

As $c$ is assumed small, we see that $V_{N L}\left(\vec{r}, \vec{r}^{\prime}\right)$ is nearly local. This is accentuated by the long-range nature of the interaction which means $v(\overrightarrow{\mathrm{q}}, \overrightarrow{\mathrm{R}})$ is peaked at $q=0$. We therefore start an expansion for $S\left(\vec{r}^{\prime}, \vec{R}\right)$ in Eq. (15) about $\vec{r}$ and ignore the higher-order derivatives. Then $V_{N L}$ can be replaced by an approximate local form $V_{L}(r)$ where

$$
V_{L}(r)=\int d^{3} r^{\prime} V_{N L}\left(\overrightarrow{\mathrm{r}}, \overrightarrow{\mathrm{r}}^{\prime}\right)=\frac{1}{R}-\frac{\operatorname{erf}\left(i^{1 / 2} \alpha+\epsilon\right)}{|\overrightarrow{\mathrm{R}}-\overrightarrow{\mathrm{r}}|} .
$$

In (16) $\alpha^{2}=|\overrightarrow{\mathrm{R}}-\overrightarrow{\mathrm{r}}|^{2} / 2 c Z$ and $\epsilon$ is a positive number to be taken in the limit to zero. The integration is performed in Appendix A.

As erf( 0$)$ is zero, there is no singularity associated with the potential at $\vec{r}=\vec{R}$, so we have removed the $\delta$-function-type singularities noted above.

The potential $V_{L}(r)$ has a particularly simple physical interpretation. A unit charge distributed with a density $\rho(0) \exp \left(-r^{2} / a^{2}\right)$ gives a static potential $\operatorname{erf}(r / a) / r$. So the effect of allowing the electron to freely recoil is to smear out the point charge.

$S$ is now given by

$$
S(\overrightarrow{\mathrm{r}} ; \overrightarrow{\mathrm{B}}, \overrightarrow{\mathrm{Z}})=\exp \left(-i c \int_{-\infty}^{Z} V_{L}\left(\overrightarrow{\mathrm{r}} ; \overrightarrow{\mathrm{B}}, \overrightarrow{\mathrm{Z}}^{\prime}\right) d Z^{\prime}\right)
$$

and in particular (see Appendix B)

$\ln S(r ; B, \infty)=-i c\left[\ln \frac{(\overrightarrow{\mathrm{B}}-\overrightarrow{\mathrm{b}})^{2}}{B^{2}}+E_{1}\left(\frac{i \alpha^{+}}{c}\right)+E_{1}\left(\frac{i \alpha^{-}}{c}\right)\right]$,

where $\alpha^{ \pm}= \pm\left[(\overrightarrow{\mathrm{B}}-\overrightarrow{\mathrm{b}})^{2}+z^{2}\right]^{1 / 2}-z$.

For large argument, the exponential integral $E_{1}(z)$ has the form $E_{1}(z) \sim e^{z} / z$. Thus, for small $c$, the $E_{1}$ terms in Eq. (17) tend to zero. We are left with just the log terms in Eq. (17) which represent the diabatic approximation solution to the prob- lem. ${ }^{6}$ This could have been obtained directly from Eq. (4). In fact, for small $c$,

$$
E_{1}\left(i \alpha^{+} / c\right) \sim\left(c / i \alpha^{*}\right) \exp \left(i \alpha^{*} / c\right),
$$

so that not only does this term decrease linearly with $c$, it also oscillates rapidly for small $c$. This will significantly decrease the effect of the correction to the diabatic approximation when our solution for $S$ is used in Eq. (9) to calculate the scattering. This is a reflection of the long-range nature of the Coulomb force we are dealing with.

For small arguments of $E_{1}(z), E_{1}(z)=\ln z-\gamma$, so if $|\vec{B}-\vec{b}|$ is small,

$$
E_{1}\left(i \alpha^{-/} / c\right)=\ln |\overrightarrow{\mathrm{B}}-\overrightarrow{\mathrm{b}}| / 2 z c-\gamma,
$$

which completely cancels the contribution to the phase coming from the electron in the diabatic approximation. Thus for large-angle scattering processes we may expect considerable differences between the diabatic and impulse approximation. This may also be true for exchange processes.

To calculate $\varphi_{s}$ in this approximation, we apply Eq. (5):

$\varphi_{s}=\exp \left(-i H_{e} Z / \hbar v\right) \varphi=\chi_{0}(r) \exp (-i \bar{H} c Z) S=\chi_{0}(r) S_{s}$.

By a similar method used above, we show

$$
\ln S_{s}=-i c \int_{-\infty}^{Z} V_{L}^{1}\left(r ; \overrightarrow{\mathrm{B}}, Z^{\prime}, Z\right) d Z^{\prime},
$$$$
\text { where } \quad V_{L}^{1}\left(r ; \overrightarrow{\mathrm{B}}, Z^{\prime}, Z\right)=\frac{\operatorname{erf}\left(i^{1 / 2} \alpha^{\prime}+E\right)}{\left|\overrightarrow{\mathrm{B}}+\overrightarrow{\mathrm{Z}}^{\prime}-\overrightarrow{\mathrm{r}}\right|}
$$

and $\quad \alpha^{\prime 2}=\left|\overrightarrow{\mathrm{B}}+\overrightarrow{\mathrm{Z}}^{\prime}-\overrightarrow{\mathrm{r}}\right|^{2} / 2 c\left(\overrightarrow{\mathrm{Z}}^{\prime}-\overrightarrow{\mathrm{Z}}\right)$

In the local impulse approximation we have neglected bt and its commutator with $\mathrm{ft}$. In Sec. III we show how to include bt to first order in $c$.

\section{BINDING TERM}

It is well known that the high-energy approximation of Glauber applied to scattering from a Coulomb force gives the exact scattering amplitude, including its phase. As the high-energy approximation, or the derivation of Eq. (3), involves neglecting a " $\nabla_{R}^{2}$ " term, it is not surprising that

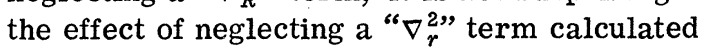
above is smaller than one might estimate from the knowledge that it was proportional to $c$. In fact, if one is considering electron-hydrogen scattering, both terms are of the same order of magnitude and should be treated together. This is not the case with bt, however, which we can expect to make a straightforward contribution proportional to $c$. This is because bt is of the form $\left[a_{0}^{-1}(\partial / \partial r)\right]$ and involves the long-range character of the force only to an inverse power while ft involves this range to an inverse-square power. So in general we can expect bt to be more important than $\mathrm{ft}$. This is a peculiarity of the Coulomb force, and it 
is fortunate that for scattering from a hydrogenlike system we can treat bt exactly.

Returning to Eq. (13), we neglect ft and its commutators with bt to give

$$
\exp (i \bar{H} c Z) \approx \exp \left(i c Z \frac{\partial}{\partial r}\right)
$$

Hence the effective potential is $V(r+i c Z ; \overrightarrow{\mathrm{B}}, Z)$, and $S$ is given by

$$
S=\exp \left[-i c \int_{-\infty}^{Z} V\left(r+i c Z^{\prime} ; B, Z^{\prime}\right) d Z^{\prime}\right] \text {. }
$$

For a potential bounded by an exponential at infinity, there is no difficulty with Eq. (19), but for the force considered here, a divergency results at large $Z$. In the limit we have

$$
\lim _{Z \rightarrow \infty} V(r+i c Z ; \overrightarrow{\mathrm{B}}, Z) \sim 1 / Z-(1 / D)(1 / Z),
$$

where

$$
\overrightarrow{\mathrm{D}}=\hat{k}-i \overrightarrow{\mathrm{c}}, \quad D=\left(1-i 2 \overrightarrow{\mathrm{c}} \cdot \hat{k}-c^{2}\right)^{1 / 2}, \quad \overrightarrow{\mathrm{c}}=c r,
$$

and $\hat{k}$ is a unit vector in the $Z$ direction.

This $Z^{-1}$ behavior at large $Z$ results in a logarithmic divergence for $S$. That our solution should be wrong at large $Z$ is not surprising, as we can see from Eq. (13). Our expansion in small $c$ is also an expansion in $Z$, and the higher-order terms which we have neglected will eventually become large and important as $Z$ increases.

If we cut off the potential at some large $Z$, i.e.,

$$
V(r, R)=0, \quad|Z|>Z_{0} \quad
$$

we find

$$
\begin{aligned}
\ln S= & -i c\left[\ln \frac{(R+Z)}{\left(B^{2}+Z_{0}^{2}\right)^{1 / 2}-Z_{0}}\right. \\
& \left.-\frac{1}{D} \ln \left(\frac{\left[(\overrightarrow{\mathrm{B}}-\overrightarrow{\mathrm{r}}+\overrightarrow{\mathrm{D}} Z)^{2}\right]^{1 / 2}+(\overrightarrow{\mathrm{B}}-\overrightarrow{\mathrm{r}}+\overrightarrow{\mathrm{D}} Z) \overrightarrow{\mathrm{D}} / D}{\left[\left(\overrightarrow{\mathrm{B}}-\overrightarrow{\mathrm{r}}-\overrightarrow{\mathrm{D}} Z_{0}\right)^{2}\right]^{1 / 2}+\left(\overrightarrow{\mathrm{B}}-\overrightarrow{\mathrm{r}}-\overrightarrow{\mathrm{D}} Z_{0}\right) \overrightarrow{\mathrm{D}} / D}\right)\right] .
\end{aligned}
$$

The $Z_{0}$ dependence of $S$ is not merely that of a harmless phase factor because the effect of bt has been to rotate the $Z$ axis into the complex plane. However, $S$ is only a slowly varying function of $Z_{0}$. Rather than leave an ambiguity in our solution, we chose a cutoff procedure which minimizes the error we have made in neglecting the higher-order commutators. This is not unique. We write

$$
\begin{aligned}
& \exp \left[i c Z \frac{\partial}{\partial r}+\frac{1}{2}\left(\frac{i c Z}{r}\right)^{2} \frac{\partial}{\partial r}+\cdots\right] \\
& \quad \approx \exp \left(i c Z \frac{\partial}{\partial r}+\frac{1}{2}(i c Z)^{2} \frac{\partial}{\partial r}+\cdots\right) \approx \exp \left(x \frac{\partial}{\partial r}\right),
\end{aligned}
$$

where $x \equiv x(Z)=\exp (i c Z)-1$,

making an error of $O(c)^{3}$ in the phase. We thus derive a potential $V(r+x, R)$ and an expression for $S$,

$$
S=\exp \left\{-i c \int_{-\infty}^{Z} V\left[r+x\left(Z^{\prime}\right) ; \overrightarrow{\mathrm{B}}, Z^{\prime}\right] d Z^{\prime}\right\},
$$

which has no divergency problems.

To include bt and ft together is now straightforward to this order in $c$.

We have

$$
S=\exp \left\{-i c \int_{-\infty}^{Z} V_{L}\left[r+x\left(Z^{\prime}\right) ; \overrightarrow{\mathrm{B}}, Z^{\prime}\right] d Z^{\prime}\right\} \text {. }
$$

To find $S_{s}$, we replace $r$ in $V_{L}^{\prime}$ by $r-x\left(Z-Z^{\prime}\right)$.

\section{CONCLUSIONS AND POSSIBLE FURTHER DEVELOPMENTS}

In Secs. I-III we have been able to show how to modify the diabatic approximation to obtain the impulse approximation and as a further refinement include the binding term to order $v^{-1}$ in terms of simple analytic expressions. The correction giving the impulse approximation is not as important in general as the binding term because it oscillates. However, in certain exchange processes this may be an important effect, as exchange integrals are small just because they have an oscillatory behavior and a stationary phase may occur. This will be left to later work. As far as the impulse approximation is concerned, we are able to treat the problem of scattering from a multielectron system in an exactly analogous way to that described above and developed by Glauber ${ }^{2}$ in the diabatic approximation. The binding term is more difficult but can always be treated in an approximate way by replacing

$$
\sum_{i} \vec{\nabla}_{i} \ln \left[\chi_{0}\left(r_{1}, r_{2}, \ldots, r_{n}\right)\right]
$$

by an appropriate constant vector.

The use of our formulas with electron scattering is a little difficult to justify as the main equation of the theory, Eq. (3), is only valid when we are already able to throw away an " $m_{p}^{-1} \nabla_{R}^{2}$ " term which will be as important as $\mathrm{ft}$ if the target particle and projectile have equal masses. However, bt may still contain an important contribution to the scattering, even with electrons as projectiles.

\section{ACKNOWLEDGMENTS}

I wish to thank Professor R. Geballe and Professor L. Wilets for showing me this interesting problem in atomic physics and to express my gratitude to Professor B. Brandsen without whose encouragement even this incomplete attempt at the problem would never have been finished.

\section{APPENDIX A}

We wish to derive Eq. (16). We have

$$
\begin{aligned}
V_{L}(r)= & \int d^{3} r^{\prime} q S\left(\overrightarrow{\mathrm{r}}-\overrightarrow{\mathrm{r}}^{\prime}+c Z \overrightarrow{\mathrm{q}}\right) \\
& \times \exp \left[\frac{1}{2} i \overrightarrow{\mathrm{q}} \cdot\left(\overrightarrow{\mathrm{r}}+\overrightarrow{\mathrm{r}}^{\prime}\right)\right] \nabla(q, R),
\end{aligned}
$$


$V_{L}(r)=\frac{1}{|\overrightarrow{\mathrm{R}}|}-\frac{2}{\pi} \frac{1}{|\overrightarrow{\mathrm{R}}-\overrightarrow{\mathrm{r}}|} \int_{0}^{\infty} \frac{\exp \left(i q^{2}\right)}{q} \sin (2 \alpha q) d q$,

where $\alpha^{2}=|\overrightarrow{\mathrm{R}}-\overrightarrow{\mathrm{r}}|^{2} / 2 c Z$.

The integral in Eq. (A2) exists for all real $a$. For $a=\infty$,

$$
V_{L}(r)=1 /|\overrightarrow{\mathrm{R}}|-1 /|\overrightarrow{\mathrm{R}}-\overrightarrow{\mathrm{r}}|
$$

Writing in Eq. (2)

$$
\frac{\sin (q 2 \alpha)}{q}=\int_{0}^{1} 2 \alpha \cos (x 2 \alpha q) d x
$$

gives on reversing the order of integration that

$$
\begin{aligned}
V_{L}(r) & =\frac{1}{R}-\frac{2}{\pi} \frac{1}{|\overrightarrow{\mathrm{R}}-\overrightarrow{\mathrm{r}}|} \int_{0}^{1} \alpha d x \exp \left(-i x^{2}\right) d x \\
& =\frac{1}{R}-\frac{2}{\sqrt{\pi|\overrightarrow{\mathrm{R}}-\overrightarrow{\mathrm{r}}|}} \int_{0}^{\exp (i \pi / 4 \alpha)} \exp \left(-t^{2}\right) d t \\
& =\frac{1}{R}-\frac{\operatorname{erf}\left(i^{1 / 2} \alpha+\epsilon\right)}{|\overrightarrow{\mathrm{R}}-\overrightarrow{\mathrm{r}}|} .
\end{aligned}
$$

We have introduced the $\epsilon$, a small positive number to be taken to zero in the limit, in order that we regain Eq. (A3) at large $\alpha$.

\section{APPENDIX B}

To prove Eq. (17) we write

$$
\begin{aligned}
& S(r ; B, \infty)=-\frac{i c}{2 \pi^{2}} \int d Z d^{3} q \\
& \quad \times \frac{\exp (-i \overrightarrow{\mathrm{q}} \cdot \overrightarrow{\mathrm{R}})-\exp \left(-i \overrightarrow{\mathrm{q}} \cdot \overrightarrow{\mathrm{R}}+i \overrightarrow{\mathrm{q}} \cdot \overrightarrow{\mathrm{r}}+\frac{1}{2} i c Z q^{2}\right)}{q^{2}} .
\end{aligned}
$$

To perform the integration, it is convenient to break the integral into two parts. However, this produces a divergency at $q=0$, so we replace " $q$ ", by " $q^{2}+m^{2}$ " in the denominator and take the limit that $m$ goes to zero at the end.

For the first integral we have

$$
\begin{aligned}
S_{1}(r ; \overrightarrow{\mathrm{B}}, \infty) & =-\frac{i c}{2 \pi^{2}} \int d Z d^{3} q \exp (i \overrightarrow{\mathrm{q}} \cdot \overrightarrow{\mathrm{R}})\left(q^{2}+m^{2}\right)^{-1} \\
& =-i c 2 K_{0}(m B) .
\end{aligned}
$$

In the limit that $m$ is zero,

$$
S_{1}(r ; B, \infty) \sim-i c 2\left(-\ln \frac{1}{2} m B-\gamma\right) .
$$

For the second integral we have

$$
\begin{aligned}
S_{2}(r ; \overrightarrow{\mathrm{B}}, \infty)= & \frac{i c}{\pi} \int d^{3} q \frac{S\left(-q \cos \theta+\frac{1}{2} c q^{2}\right)}{\left(q^{2}+m^{2}\right)} \\
& \times \exp [-i q \cdot(B-b) \sin \theta \cos \varphi-i q z \cos \theta] \\
= & 2 i c \int_{0}^{\pi / 2} \frac{d \theta \sin \theta \cos }{\sin ^{2} \theta+\frac{1}{4} c^{2} m^{2}} J_{0} \\
& \times\left(\frac{2}{c} \sin \theta \cos \theta|\overrightarrow{\mathrm{B}}-\overrightarrow{\mathrm{b}}|\right) \exp \left[(i 2 z / c) \sin ^{2} \theta\right] .
\end{aligned}
$$

If we write

$$
g(p)=\int_{0}^{\infty} \exp \left(-r^{2} p\right) d\left(r^{2}\right) \int_{0}^{\pi / 2} F(\theta, r) d \theta,
$$

we note that $\int_{0}^{\pi / 2} F(\theta, r) d \theta$ is the inverse Laplace transform of $g(p)$. Hence the inverse Laplace transform of the integral we require is given by

$$
\begin{aligned}
g(p)= & 4 i c \int_{0}^{\infty} d x \int_{0}^{\infty} d y \frac{\exp \left(-x^{2} p-y^{2} p+2 i y^{2} z / c\right)}{\left(y^{2}+c^{2} m^{2} / 4\right)} \\
& \times J_{0}(y x|\overrightarrow{\mathrm{B}}-\overrightarrow{\mathrm{b}}| 2 / c) \\
= & \frac{i c}{p} \int_{c^{2} m^{2} / 4}^{\infty} \exp \left[\left(\frac{m^{2} c^{2}}{4}-y\right)\right. \\
& \left.\times\left(p-\frac{2 i z}{c}+\frac{(\overrightarrow{\mathrm{B}}-\overrightarrow{\mathrm{b}})^{2}}{c^{2} p}\right)\right] \frac{d y}{y} \\
= & \frac{i c}{p} \exp \left(\frac{c^{2} m^{2} p}{4}-\frac{i m^{2} c z}{2}+\frac{(\overrightarrow{\mathrm{B}}-\overrightarrow{\mathrm{b}})^{2} m^{2}}{4 p}\right) \\
& \times E_{1}\left(\frac{c^{2} m^{2} p}{4}-\frac{i m^{2} c z}{2}+\frac{(\overrightarrow{\mathrm{B}}-\overrightarrow{\mathrm{b}})^{2} m^{2}}{4 p}\right) .
\end{aligned}
$$

In the limit $m$ is zero this gives

$$
g(p)=\frac{i c}{p}\left[-\gamma-\ln \left(\frac{c^{2} m^{2} p}{4}-\frac{i m^{2} c z}{2}+\frac{(\overrightarrow{\mathrm{B}}-\overrightarrow{\mathrm{b}})^{2} m^{2}}{4 p}\right)\right] .
$$

The Laplace transform of the exponential integral $E_{1}(z)$,

$$
E_{1}(z)=\int_{1}^{\infty} \frac{\exp (-z x)}{x} d x
$$

is given by

$e_{1}(p)=\int_{0}^{\infty} \exp (-p t) E_{1}(\alpha+t) d t=p^{-1}[\ln (\alpha+p)-\ln \alpha]$.

The Laplace transfer of $(\gamma+\ln t)$ is similarly shown to be $\left(-p^{-1} \ln p\right)$.

Hence the inverse Laplace transform of $g(p)$ is given by

$$
\begin{aligned}
& \begin{array}{l}
f(t)=i c\left[-2 \gamma-2 \ln \frac{1}{2} m+E_{1}\left(\frac{i \alpha^{*} t}{c}\right)\right. \\
\left.+\left(\frac{i \alpha^{-} t}{c}\right)-\ln t(\overrightarrow{\mathrm{B}}-\overrightarrow{\mathrm{b}})^{2}\right],
\end{array} \\
& \text { where } \alpha^{+}=+\left[(\overrightarrow{\mathrm{B}}-\overrightarrow{\mathrm{b}})^{2}+z^{2}\right]^{1 / 2}-z \\
& \text { and } \quad \alpha^{-}=-\left[(\overrightarrow{\mathrm{B}}-\overrightarrow{\mathrm{b}})^{2}+z^{2}\right]^{1 / 2}-z .
\end{aligned}
$$

We need $f(t)$ where

$$
f(t)=\int_{0}^{\pi / 2} F(\theta, \sqrt{t}) d \theta
$$

where $t$ is unity. Hence, $S(r ; \overrightarrow{\mathrm{B}}, \infty)=-i c\left[\ln \frac{(\overrightarrow{\mathrm{B}}-\overrightarrow{\mathrm{b}})^{2}}{B^{2}}+E_{1}\left(\frac{i \alpha^{+}}{c}\right)+E_{1}\left(\frac{i \alpha^{-}}{c}\right)\right]$. 
* Presented at the Tenth North Eastern Universities Symposium on Atomic Physics, Durham, United Kingdom, 1968 (unpublished).

$\dagger$ Part of this work was carried out while the author was a fellow at the Atomic Energy Research Establishment, Harwell, U. K. Work supported by a grant from the National Science Foundation.

${ }^{1}$ This approximation has been called elsewhere in the literature the sudden, the adiabatic, and the quasielastic approximation [J. F. Reading and Alan D. MacKellar, Phys. Rev. 173, 1026 (1968); T. Tamura, Rev. Mod. Phys. 37, 679 (1965); M. L. Goldberger and K. M. Watson, Collision Theory (Wiley, New York, 1964)].

${ }^{2} \mathrm{R}$. Glauber, in Lectures in Theoretical Physics, edited by W. E. Brittin et al. (Interscience, New York, 1958), Vol. II; M. Mittleman, Phys. Rev. 122, 499 (1961).
${ }^{3}$ This program has been carried out with some success by Mittleman and Quong for short range forces [M. H. Mittleman and J. Quong, Phys. Rev. 167, 74 (1968)].

${ }^{4}$ H. F. Baker, Proc. London Math. Soc. 2, Series 13 , 24 (1905); W. A. Magnus, Ann. Math. 52, 111 (1950).

${ }^{5}$ K. M. Watson, Phys. Rev. 89,575 (1953). See also Goldberger and Watson, Ref. 1, for detailed references. Applications for electron-hydrogen have been given by Akerib and Borowitz [R. Akerib and S. Borowitz, Phys. Rev. 122, (1961)]. Proton-hydrogen scattering has been studied in the impulse approximation by Cheshire [I. M. Cheshire, Proc. Phys. Soc. (London) 82, 113 (1963)].

${ }^{6}$ See for example an application to electron-hydrogen scattering by V. Franco, Phys. Rev. Letters 20, 709 (1968).

\title{
Second-Order Calculations of the Single-Quantum Annihilation Cross Sections in Various Atomic Shells*广
}

\author{
David S. Moroi, Andreas Davidz, and Rudolph F. Kess \\ Department of Physics, Kent State University, Kent, Ohio 44240 \\ (Received 15 August 1969)
}

\begin{abstract}
A general method for calculating the cross sections for single-quantum annihilation (SQA) of positrons in various atomic shells, valid to second order in the $\alpha Z$ expansion (one order beyond the leading term), is presented with the help of the modified Sommerfeld-Maue wave function for incoming positrons and the relativistic wave functions for the bound electrons in any atomic shells. It is shown in this approximation that the differential cross sections are of the form $\sigma_{n j l}(k, q)=C_{n l} \sigma_{n_{0} j l}(k, q)$, where $n_{0}=l+1$, and $k$ and $q$ are the photon energy and the magnitude of momentum transfer to a nucleus, respectively: The factor $C_{n l}$ is evaluated in general. The SQA cross sections are calculated for the $1 s_{1 / 2}, 2 p_{1 / 2}$, and $2 p_{3 / 2}$ states to 2 nd order in the $\alpha Z$ expansion and for the $3 d_{3 / 2}$ and $3 d_{5 / 2}$ states to lowest order in the $\alpha Z$ expansion, and are in closed form. The relationship between the SQA cross section for any state and the corresponding cross section for the atomic photoeffect is discussed. The leading term for the SQA cross section is closely related to that for the photoeffect, but there is a distinct difference between the correction terms for the former and the latter. The comparison of the results of the present work for the $K$ and $L_{\mathrm{I}}$ shells with a recent numerical calculation done by others shows that for low and intermediate energies, the above factorization rule is valid within $1.4 \%$ for $Z=47$ and $3.8 \%$ for $Z=90$. However, the $1 s_{1 / 2}$ cross section for $Z \geqq 82$ is negative for any energy below $2.264 \mathrm{Mev}$.
\end{abstract}

\section{INTRODUCTION}

The purposes of this paper are twofold: first, to calculate in closed analytical form the second-order cross sections for the single-quantum annihilation (SQA) of positrons with bound atomic electrons in several atomic shells, with the help of the modified Sommerfeld-Maue wave functions ${ }^{1}$ for the ingoing positrons; second, to study the relationship between the above cross sections and those for the atomic photoeffect. ${ }^{2,3}$

The numerical calculations of the SQA cross sections for the $K$ shell were done first by Jaeger and Hulme, ${ }^{4}$ later by Johnson, Buss and Carrol, ${ }^{5}$ and more recently by Johnson. ${ }^{6}$ Sheth and Swamy ${ }^{7}$ used numerical techniques to calculate the SQA cross section for the $L_{1}$ shell. The third-order calculation of the SQA cross section for the $K$ shell was carried by Carroll and O'Connell. ${ }^{B}$ This last result for the SQA cross section, however, was not in a closed form.

One can, however, calculate the second-order SQA cross sections for arbitrary shells in closed analytical form (polynomials in momenta and energy). It is, therefore, instructive to obtain the 